\title{
Hematological Malignancies in the Omics Era
}

\section{Ramzi M. Mohammad ${ }^{\text {** }}$ and Asfar S. Azmi ${ }^{2}$}

${ }^{1}$ Department of Oncology, Karmanos Cancer Institute, USA

${ }^{2}$ Department of Pathology, Wayne State University, Detroit MI, 48201, USA

Hematological malignancies are a collective term for a group of blood cancers originating from bone marrow or lymph. Even though there are some improvements in understanding the biology of hematologic malignancies and the development of new therapies are occurring at a rapid pace, a lot of work is still needed. Standard chemotherapy did not do much to change the dismal survival statistics and therapies that target single molecules have not resulted in expected outcome (except in few cases like chronic myelognous leukemia CML) with majority of patients dying from recurring disease. Nevertheless, in the past few years the field has witnessed a number of key advancements in our understanding of these deadly malignancies that has led to the development of a newer combination and targeted therapies [1]. For example the identification of molecular pathophysiology of the BCRABL and studies of the mechanisms of resistance in Philadelphia chromosome $(\mathrm{Ph})$-positive chronic myeloid leukemia resulted in the rapid development of multiple selective BCR-ABL tyrosine kinase inhibitors that has dramatically improved 10-year survival from approximately $20 \%$ to an estimated rate of $85 \%$ [2]. Nevertheless, development of resistance to treatment is a recurring phenomenon and we are still a long way from conquering the disease [3]. Major challenges include the incomplete understanding and validation of newly identified targets in patients, the multiplicity and complexity of genetic and epigenetic changes and the redundancies and cross-talk found in key signaling pathways. Another level of complexity comes from regulatory mechanisms emanating from non-coding RNAs (miRNAs) that are also under epigenetic regulatory mechanisms [4]. To overcome these barriers, it is important to understand the molecular cross-talk among key signaling pathways in hematological malignancies and how they may be altered by targeted agents. Innovative approaches are definitely needed, such as understanding the global physiologic environment of target proteins and the effects of modifying them without losing key molecular details. Such strategies will aid the design of novel therapeutics and their combinations against this multifaceted disease, in which efficacious combination therapies will focus on altering multiple pathways rather than single proteins.

Post human genome project we have the availability of large datasets obtained from different sources such as expression profiles pre- and post therapeutic treatments from cured and recurring diseased patients as well as profiles of different cancer sub-types. Probing these available information can help in deeper understanding of the disease. When investigated the right way, these expression datasets can provide key information to weaker links in the complex disease pathway that can provide avenues for novel treatments. However, traditional molecular biology has its limitations and can only peripherally help in unweaving this complex web of information. There are advanced and emerging technologies such as systems biology and molecular network modeling that can help in stratifying the vast available datasets into biologically meaningful information. Few groups have already utilized these high throughput technologies to investigate the redundant expression patterns in large patient datasets [5]. From these meaningful patterns researchers are now able to extract important information on weak links or druggable avenues using available and easy to use gene ontology programs [6]. The technologies have also been utilized in gathering patient specific information that is expected to aid the development of individualized therapies.

A major thrust of research has centered on the role of miRNAs in the development of hematological malignancies, as well as in drug resistance. Intense research on this topic has revealed that miRNA alterations are involved in the initiation and progression of blood cancers [7]. The causes of such widespread differential expression of miRNA genes in malignant compared with normal cells can partially be explained by the location of these genes in cancer-associated genomic regions, by epigenetic mechanisms and by alterations in the miRNA processing machinery. Additionally, miRNA-expression profiling of human tumors has identified signatures associated with diagnosis, staging, progression, prognosis and response to treatment. MiRNAs appear particularly suitable to serve as hubs of regulatory networks underlying complex diseases. However, it remains unknown whether the set of miRNA target genes designated are regulated by an individual miRNA constituting the biological network of functionally-associated molecules or reflects a random set of functionally-independent genes. Prior to clinical application of miRNA as therapeutics for hematological malignancies, clear experimental evidence for coordinated regulation of a large number of genes by miRNAs that includes the entire set of on and off target pathways is absolutely necessary. The complexity of the action of miRNAs calls for comprehensive, integrative systems approaches to examine the effect of these miRNAs in their entirety. Here too systems biology can be helpful in addressing clinically relevant questions. Notably, advanced proteomic techniques have begun to be utilized in the analysis of widespread effects of miRNAs. Other approaches that have been used include large scale sequencing of miRNA and potential miRNA targets, mRNA expression profiling, and bioinformatic modeling. Systems technologies can help in mining large diagnostic and prognostic datasets, can rapidly translate complex miRNA target interactomes and can aid in the identification of target patient population that will most effectively respond to any newly designed miRNA based therapy.

Presently we are in the omics era where cutting edge and high throughput data management technologies are available that can rapidly manage and simplify large datasets into meaningful information. It is upto the research community how it utilizes these cutting edge technologies to decipher unanswered problems in blood cancer. This

*Corresponding author: Ramzi M. Mohammad, Department of Oncology, Karmanos Cancer Institute, Wayne State University, USA, Tel: +313-576-8329; Fax: +313-576-8389; E-mail: mohammar@karmanos.org

Received December 22, 2011; Accepted January 02, 2012; Published January 04, 2012

Citation: Mohammad RM, Azmi AS (2012) Hematological Malignancies in the Omics Era. J Blood Lymph 2:e106. doi:10.4172/2165-7831.1000e106

Copyright: () 2012 Mohammad RM, et al. This is an open-access article distributed under the terms of the Creative Commons Attribution License, which permits unrestricted use, distribution, and reproduction in any medium, provided the original author and source are credited. 
would certainly require a departure from protein centric to a more pathway centric approach. When combined, traditional molecular biology and systems biology can accelerate understanding of blood cancer and can drive the rapid development therapeutic strategies incorporating newer combinations regimens personalized cancer medicine.

\section{References}

1. Kantarjian $\mathrm{H}$, Schiffer $\mathrm{C}$, Burnett $\mathrm{A}$ (2011) Hematologic malignancies: where do we stand in 2011? J Clin Oncol 29: 473-474.

2. SmithCC, Shah NP (2011) Tyrosine kinase inhibitor therapy for chronic myeloid leukemia: approach to patients with treatment-naive or refractory chronicphase disease. Hematology Am Soc Hematol Educ Program 2011: 121-127.
3. Devita VT Jr, Canellos GP (2011) Hematology in 2010: New therapies and standard of care in oncology. Nat Rev Clin Oncol 8: 67-68.

4. Fatica A, Bozzoni I (2009) Role of microRNAs in hematological malignancies Expert Rev Hematol 2: 415-423.

5. Zamo A, Cecconi D (2010) Proteomic analysis of lymphoid and haematopoietic neoplasms: there's more than biomarker discovery. J Proteomics 73: 508-520.

6. Tian Q, Price ND, Hood L (2011) Systems Cancer Medicine: Towards Realization of Predictive, Preventive, Personalized, and Participatory (P4) Medicine. J Intern Med 271: 111-121.

7. Moussay E, Wang K, Cho JH, van Moer K, Pierson S, et al. (2011) MicroRNA as biomarkers and regulators in B-cell chronic lymphocytic leukemia. Proc Natl Acad Sci U S A 108: 6573-6578. 